\title{
Calculating Intrinsic and Extrinsic Camera Parameters Based on the PnP Problem
}

\author{
Yue Zhao, Yalin Wang \& Qian Cui \\ School of Mathematics and Statistics, Yunnan University \\ Cuihu North Road 2\#, Kunming, 650091, China \\ Email: zhao6685@yeah.net
}

\begin{abstract}
The classical PnP problem is premised on given intrinsic camera parameters. However, for unknown intrinsic camera parameters, given $n$ space points in a world coordinate system and their coordinates in an image coordinate system, the extrinsic camera parameters can be determined. Regarding the existence and uniqueness of a solution for the classical PnP problem, for 4 control points in a plane and an uncalibrated camera, a set of linear equations can be solved based on the correspondence between the space points and the image points. The results show that this approach is feasible and has high calculation precision.
\end{abstract}

Keywords: computer vision; extrinsic parameters; intrinsic parameters; PnP problem.

\section{$1 \quad$ Introduction}

The PnP problem is a classical problem not only in computer vision and photogrammetry but also in mathematics. Since this problem was first proposed by Fischler and Bolles [1] in 1981, it has attracted considerable attention because of its high value in object pose estimation. And it has been fully tested with the emergence of further literature [1-3]. For cases where the intrinsic camera parameters are known, there has been extensive study of the P3P $[4,5]$, $\mathrm{P} 4 \mathrm{P}$ and P5P $[6,7]$ problems. The P3P problem has at most 4 solutions and this upper bound is attainable. The $\mathrm{P} 4 \mathrm{P}$ problem has a unique solution when the 4 control points are coplanar. If the 4 control points are non-coplanar, there may be at most 4 solutions, of which the upper bound is also attainable. The P5P problem has at most 2 solutions and this upper bound is also attainable. When the intrinsic camera parameters are unknown, the camera's intrinsic and extrinsic parameters in the world coordinate system (WCS) can be solved under the condition that the coordinates of $\mathrm{n}$ points are known in both the WCS and the image coordinate system (ICS), which is called the uncalibrated PnP problem. In practical problems, such as tasks involving active vision, the intrinsic camera parameters must frequently adjust and change, so it cannot be assumed that the camera parameter matrix is known. Therefore, the uncalibrated PnP problem is highly significant. When there are 5 control points in which any 4 points are non-coplanar or any 3 points are non-collinear [6-8], there are two

Received July $22^{\text {nd }}, 2013$, Revised February $4^{\text {th }}, 2014$, Accepted for publication May $7^{\text {th }}, 2014$

Copyright (C) 2014 Published by ITB Journal Publisher, ISSN: 2337-5779, DOI: 10.5614/j.eng.technol.sci.2014.46.3.2 
possibilities regarding the uncalibrated $\mathrm{P} 5 \mathrm{P}$ problem: at most 4 solutions or infinitely many solutions. Therefore, it is difficult to solve for the intrinsic and extrinsic parameters in the uncalibrated P5P problem [8-10].

In our study, we have used the $\mathrm{P} 4 \mathrm{P}$ problem to solve the intrinsic and extrinsic camera parameters and a frustum of a regular hexagonal pyramid was used as a calibration block.

\section{$2 \quad$ Pinhole Camera Model}

The camera model adopted in our study is the pinhole model of optical imaging in ideal conditions (see Figure 1) [11,12].

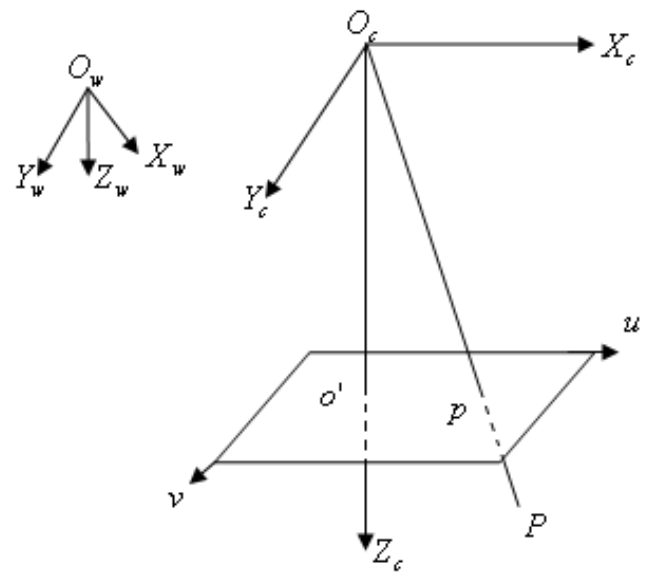

Figure 1 Camera imaging model.

Given a point $P$ in space, the point $P$ in the $\operatorname{WCS}\left(O_{w}-X_{w} Y_{w} Z_{w}\right)$ is denoted as $P=\left(x_{w}, y_{w}, z_{w}\right)$, of which the image coordinate is $p=(u, v)$. Based on the imaging principle, a point in $3 \mathrm{D}$ space can be obtained on the image plane:

$$
\lambda\left[\begin{array}{l}
u \\
v \\
1
\end{array}\right]=K\left[\begin{array}{ll}
R & t
\end{array}\right]\left[\begin{array}{c}
x_{w} \\
y_{w} \\
z_{w} \\
1
\end{array}\right]=\left[\begin{array}{ccc}
f_{x} & s & u_{0} \\
0 & f_{y} & v_{0} \\
0 & 0 & 1
\end{array}\right]\left[\begin{array}{llll}
r_{11} & r_{12} & r_{13} & t_{1} \\
r_{21} & r_{22} & r_{23} & t_{2} \\
r_{31} & r_{32} & r_{33} & t_{3}
\end{array}\right]\left[\begin{array}{c}
x_{w} \\
y_{w} \\
z_{w} \\
1
\end{array}\right]
$$

where $\lambda$ refers to the nonzero scale factor; $K$ is called the matrix of the intrinsic camera parameters; $\left(u_{0}, v_{0}\right)$ is the principle point; $s$ is the distortion factor; $f_{x}, f_{y}$ represent the focal length in the direction of the $u$ axis and the $v$ axis, 
respectively; $\left(r_{i j}\right)$ is the rotation matrix of $3 \times 3$ units; and $\left(t_{1}, t_{2}, t_{3}\right)^{T}$ is the translation vector. A regular prismoid is used as a calibration block. Without loss of generality, the plane on which the space points are located in the WCS is defined as the $O_{w} X_{w} Y_{w}$ plane (see Figure 1), so that Eq. (1) can be simplified as follows:

$$
\lambda\left[\begin{array}{c}
u \\
v \\
1
\end{array}\right]=\left[\begin{array}{ccc}
f_{x} & s & u_{0} \\
0 & f_{y} & v_{0} \\
0 & 0 & 1
\end{array}\right]\left[\begin{array}{lll}
r_{11} & r_{12} & t_{1} \\
r_{21} & r_{22} & t_{2} \\
r_{31} & r_{23} & t_{3}
\end{array}\right]\left[\begin{array}{c}
x_{w} \\
y_{w} \\
z_{w} \\
1
\end{array}\right]
$$

\section{Determining the Extrinsic Camera Parameters in the Classical PnP Problem}

In the classical PnP problem, it is assumed that the camera model is the classical pinhole imaging model and the matrix of the intrinsic camera parameters is given. An image containing $\mathrm{n}$ points is captured and the coordinates of the $\mathrm{n}$ points are given in the WCS and the ICS. The direction and position $\left[\begin{array}{ll}R & t\end{array}\right]$ of the camera can be obtained, in which $R$ represents the rotation matrix to indicate the direction of the camera and $t$ is the translation vector to indicate the position of the camera.

By using the uniqueness of the solution to the PnP problem, the extrinsic camera parameters can be obtained. The matrix $K$ of the intrinsic camera parameters is known based on the given 4 coplanar points $P_{i}=\left(x_{i}, y_{i}, z_{i}\right)^{T}$ in space and their corresponding points $p_{i}=\left(u_{i}, v_{i}\right)^{T}$ in the image. The plane of the coplanar points in the WCS is defined as the $O_{w} X_{w} Y_{w}$ plane (see Figure 1), and the coordinates of the coplanar points in the WCS can be rewritten as $P_{i}=\left(x_{i}, y_{i}, 0\right)^{T}, i=1,2,3,4$, so Eq. (3) can be derived from Eq. (2) as follows:

$$
\lambda_{i}\left(\begin{array}{c}
u_{i} \\
v_{i} \\
1
\end{array}\right)=K\left(\begin{array}{lll}
r_{1} & r_{2} & t
\end{array}\right)\left(\begin{array}{c}
x_{i} \\
y_{i} \\
1
\end{array}\right), i=1,2,3,4
$$

in which $K,\left(\begin{array}{ll}x_{i} & y_{i}\end{array}\right)^{T},\left(\begin{array}{ll}u_{i} & v_{i}\end{array}\right)^{T}(i=1,2,3,4)$ are the known quantities. Because $r_{3}=r_{1} \times r_{2}$, the extrinsic camera parameters can be used to solve for $r_{1}, r_{2}, t$. 
Let $M=\left[\begin{array}{lll}r_{11} & r_{12} & t_{1} \\ r_{21} & r_{22} & t_{2} \\ r_{31} & r_{23} & t_{3}\end{array}\right]=K\left(\begin{array}{lll}r_{1} & r_{2} & t\end{array}\right)$. Then, Eq. (3) becomes

$$
\lambda_{i}\left(\begin{array}{c}
u_{i} \\
v_{i} \\
1
\end{array}\right)=M\left(\begin{array}{c}
x_{i} \\
y_{i} \\
1
\end{array}\right)=\left[\begin{array}{lll}
r_{11} & r_{12} & t_{1} \\
r_{21} & r_{22} & t_{2} \\
r_{31} & r_{23} & t_{3}
\end{array}\right]\left(\begin{array}{c}
x_{i} \\
y_{i} \\
1
\end{array}\right), i=1,2,3,4
$$

According to Eq. (4), each point in space provides two constraints related to $r_{i j}(i, j=1,2,3)$ and $t_{i}(i=1,2,3)$; therefore, 4 points provide 8 homogeneous linear equations related to $r_{i j}(i, j=1,2,3)$ and $t_{i}(i=1,2,3)$, and thus $M$ can be uniquely determined up to a constant factor. The relationship between the projection matrix $M$ and $r_{1}, r_{2}, t$ can be expressed as follows:

$$
s M=K\left(\begin{array}{lll}
r_{1} & r_{2} & t
\end{array}\right)
$$

or

$$
\left(\begin{array}{lll}
r_{1} & r_{2} & t
\end{array}\right)=s K^{-1} M
$$

where $s$ is the non-zero invariant factor.

Because the rotation matrix $R$ is a unit orthogonal matrix, it has $\left|r_{1}\right|=\left|r_{2}\right|=1$, $r_{3}=r_{1} \times r_{2}$. According to $\left|r_{1}\right|=\left|r_{2}\right|=1$ and Eq. (6), the invariant factor $s$ can be obtained and then $r_{1}, r_{2}, t$ can be determined uniquely, which makes it possible to solve for $r_{3}=r_{1} \times r_{2}, R$ and $t$.

\section{Solving for the Intrinsic and Extrinsic Parameters Using the Classical PnP Problem}

In the PnP problem for an uncalibrated camera, the intrinsic camera parameters are unknown and $n$ points must be known in the WCS and the ICS to solve for the intrinsic and extrinsic camera parameters in the WCS. Because it is difficult to solve for the intrinsic and extrinsic camera parameters in the uncalibrated $\mathrm{P} 5 \mathrm{P}$ problem, we present the results of our research on the uncalibrated $\mathrm{P} 5 \mathrm{P}$ problem below. 


\subsection{Solving Projection Matrix}

Take 4 arbitrary points $P_{i}=\left(x_{i}, y_{i}, z_{i}\right)^{T} \quad(i=1,2,3,4)$ and their corresponding image points $p_{i}=\left(u_{i}, v_{i}\right)^{T}(i=1,2,3,4)$, denoted as $\bar{P}_{i}=\left(x_{i}, y_{i}, z_{i}, 1\right)^{T}$ and $\bar{p}_{i}=\left(u_{i}, v_{i}, 1\right)^{T}$, respectively. From Eq. (2), we obtain:

$$
\lambda_{i} \bar{p}_{i}=H \bar{P}_{i}, \quad i=1,2,3,4
$$

Let the camera projection matrix be $H=\left[\begin{array}{llll}r_{11} & r_{12} & r_{13} & t_{1} \\ r_{21} & r_{22} & r_{23} & t_{2} \\ r_{31} & r_{32} & r_{33} & t_{3}\end{array}\right]$. Each point in space can provide two constraints related to $r_{i j}(i, j=1,2,3)$ and $t_{i}(i=1,2,3)$, and therefore 4 points can provide eight homogeneous linear equations related to $r_{i j}(i, j=1,2,3)$ and $t_{i}(i=1,2,3)$. When the 4 points are non-coplanar, $H$ cannot be determined because there are 12 parameters in $H$. When the four are coplanar, without loss of generality, the plane where they are located is defined as the $O_{w} X_{w} Y_{w}$ plane in the WCS and the coordinates of the point in the WCS can be modified to $P_{i}=\left(x_{i}, y_{i}, 0\right)^{T}$, allowing Eq. (7) to be rewritten as follows:

$$
\lambda_{i}\left(\begin{array}{c}
u_{i} \\
v_{i} \\
1
\end{array}\right)=M\left(\begin{array}{c}
x_{i} \\
y_{i} \\
1
\end{array}\right), \quad i=1,2,3,4
$$

where $M=\left[\begin{array}{lll}r_{11} & r_{12} & t_{1} \\ r_{21} & r_{22} & t_{2} \\ r_{31} & r_{23} & t_{3}\end{array}\right]=K\left(\begin{array}{llll}r_{1} & r_{2} & t\end{array}\right) ; r_{1}, r_{2}, r_{2}$ are three column vectors of the rotation matrix $R$; and $r_{3}=r_{1} \times r_{2}$. Thus, 4 points provide 8 homogeneous linear equations related to $M$, and $M$ can be uniquely determined up to a constant factor.

\subsection{Solving for Intrinsic and Extrinsic Camera Parameters}

\subsubsection{Solving for Intrinsic Parameters}

Our study expresses the camera imaging model using the well-known pinhole camera model. In Eq. (1), let the distortion factor $s$ be 0 . Then the intrinsic matrix is as follows: 


$$
K=\left[\begin{array}{ccc}
f_{x} & 0 & u_{0} \\
0 & f_{y} & v_{0} \\
0 & 0 & 1
\end{array}\right]
$$

To solve for the camera intrinsic and extrinsic parameters, first, suppose the principal point is located at the origin of the ICS. Then, $f_{x}, f_{y}$ can be solved by the linear least square method and the coordinates $\left(u_{0}, v_{0}\right)$ of the principal point can be determined based on $f_{x}, f_{y}$. The extrinsic camera parameters can then be determined using the character of the homography matrix and the rotation matrix. For ease of calculation, the intrinsic camera parameters are simply decomposed into

$$
K=\left[\begin{array}{ccc}
f_{x} & 0 & u_{0} \\
0 & f_{y} & v_{0} \\
0 & 0 & 1
\end{array}\right]=\left[\begin{array}{ccc}
1 & 0 & u_{0} \\
0 & 1 & v_{0} \\
0 & 0 & 1
\end{array}\right]\left[\begin{array}{ccc}
f_{x} & 0 & 0 \\
0 & f_{y} & 0 \\
0 & 0 & 1
\end{array}\right]
$$

Let

$$
K_{1}=\left[\begin{array}{ccc}
1 & 0 & u_{0} \\
0 & 1 & v_{0} \\
0 & 0 & 1
\end{array}\right], K_{2}=\left[\begin{array}{ccc}
f_{x} & 0 & 0 \\
0 & f_{y} & 0 \\
0 & 0 & 1
\end{array}\right]
$$

where $K_{1}, K_{2}$ are invertible matrices. To solve $f_{x}, f_{y}$, suppose the principal point of an image is located at the origin of the ICS. If the resolution of the camera is $c_{1} \times c_{2}$, the coordinates of the principal point are $\left(u_{0}, v_{0}\right)^{T}=\left(\frac{c_{1}}{2}, \frac{c_{2}}{2}\right)^{T}$, or $u_{0}=\frac{c_{1}}{2}, v_{0}=\frac{c_{2}}{2}$, so $K_{1}$ can be determined. As $M=K\left(\begin{array}{lll}r_{1} & r_{2} & t\end{array}\right)$ $=K_{1} K_{2}\left(\begin{array}{lll}r_{1} & r_{2} & t\end{array}\right)$ is known, both sides of the equation can be multiplied by $K_{1}^{-1}$ to obtain Eq. (12).

$$
K_{1}^{-1} M=K_{2}\left(\begin{array}{lll}
r_{1} & r_{2} & t
\end{array}\right)
$$

Let $L=K_{1}^{-1} M$ and then

$$
L=K_{2}\left(\begin{array}{lll}
r_{1} & r_{2} & t
\end{array}\right)
$$

After multiplying both sides of Eq. (13) by $K_{2}^{-1}$, we can rearrange it to obtain

$$
r_{1}=K_{2}^{-1} l_{1}, r_{2}=K_{2}^{-1} l_{2}
$$


The rotation matrix $R$ is a unit orthogonal matrix, and $r_{1}^{T} r_{2}=0,\left|r_{1}\right|=\left|r_{2}\right|=1$, from which two constraint equations can be obtained:

$$
\begin{aligned}
& l_{1}^{T} K_{2}^{-T} \cdot K_{2}^{-1} l_{2}=0 \\
& l_{1}^{T} K_{2}^{-T} \cdot K_{2}^{-1} l_{1}=l_{2}^{T} K_{2}^{-T} \cdot K_{2}^{-1} l_{2}
\end{aligned}
$$

The expansion of Eq. (15) is as follows:

$$
\begin{aligned}
& \frac{l_{11} l_{12}}{f_{x}^{2}}+\frac{l_{21} l_{22}}{f_{y}^{2}}+l_{31} l_{32}=0 \\
& \frac{l_{11}^{2}}{f_{x}^{2}}+\frac{l_{21}^{2}}{f_{y}^{2}}+l_{31}^{2}=\frac{l_{12}^{2}}{f_{x}^{2}}+\frac{l_{22}^{2}}{f_{y}^{2}}+l_{32}^{2}
\end{aligned}
$$

Eq. (16) can be rearranged as follows:

$$
\left(\begin{array}{cc}
l_{11} l_{12} & l_{21} l_{22} \\
l_{11}^{2}-l_{12}^{2} & l_{21}^{2}-l_{22}^{2}
\end{array}\right)\left(\begin{array}{l}
1 / f_{x}^{2} \\
1 / f_{y}^{2}
\end{array}\right)=\left(\begin{array}{c}
-l_{31} l_{32} \\
-\left(l_{31}^{2}-l_{32}^{2}\right)
\end{array}\right)
$$

From Eq. (17), each image can provide two constraint equations related to $f_{x}, f_{y}$. Based on knowing $f_{x}>0, f_{y}>0, f_{x}, f_{y}$ can be calculated. After $f_{x}, f_{y}$ have been determined, two constraint equations can be solved in the same way using the unit orthogonal matrix $R$ :

$$
\begin{aligned}
& m_{1}^{T} K^{-T} K^{-1} m_{2}=0 \\
& m_{1}^{T} K^{-T} K^{-1} m_{1}=m_{2}^{T} K^{-T} K^{-1} m_{2}
\end{aligned}
$$

In Eq. (18), $m_{1}, m_{2}$ denote the first two columns of matrix $M$, respectively. Eq. (18) can be expanded and rearranged as follows:

$$
\left(\begin{array}{ccc}
\frac{r_{31} r_{12}+r_{11} r_{32}}{f_{x}^{2}} & \frac{r_{11} r_{22}+r_{21} r_{32}}{f_{y}^{2}} & -r_{31} r_{32} \\
\frac{2\left(r_{31} r_{11}-r_{12} r_{32}\right)}{f_{x}^{2}} & \frac{2\left(r_{21} r_{31}-r_{22} r_{32}\right)}{f_{y}^{2}} & -\left(r_{31}^{2}-r_{32}^{2}\right)
\end{array}\right)\left(\begin{array}{c}
u_{0} \\
v_{0} \\
\frac{u_{0}^{2}}{f_{x}^{2}}+\frac{v_{0}^{2}}{f_{y}^{2}}
\end{array}\right)=\left(\begin{array}{c}
\frac{r_{11} r_{12}}{f_{x}^{2}}+\frac{r_{21} r_{22}}{f_{y}^{2}}+r_{31} r_{32} \\
\frac{r_{11}^{2}-r_{12}^{2}}{f_{x}^{2}}+\frac{r_{21}^{2}-r_{22}^{2}}{f_{y}^{2}}+r_{31}^{2}-r_{32}^{2}
\end{array}\right)
$$

As mentioned above, Eq. (18) can provide two constraints related to $u_{0}, v_{0}$ in each image and therefore, $u_{0}, v_{0}$ can be solved. 


\subsubsection{Solving for Extrinsic Parameters}

Because $f_{x}, f_{y}, u_{0}, v_{0}$ can be solved as discussed above, the intrinsic camera parameter matrix is known. We can then use the classical PnP problem to solve for the extrinsic camera parameters, as described in Section 3.

From the above discussion, we obtain the following algorithm:

Take a regular hexagonal prismoid as a calibration block to determine the camera's intrinsic and extrinsic parameters.

Step 1: Each point in the WCS provides 2 constraints related to $r_{i j}(i, j=1,2,3)$ and $t_{i}(i=1,2,3)$, so $M$ can be determined using 4 points. According to Eq. (5), Eq. (6) and $\left|r_{1}\right|=\left|r_{2}\right|=1, s$ can be calculated, and the extrinsic parameters can also be obtained.

Step 2: According to Eq. (19), each image can provide two constraints related to $u_{0}, v_{0}$, so $u_{0}, v_{0}$ can be calculated using three images.

Step 3: Each image can provide two constraints related to $f_{x}, f_{y}$, so $f_{x}, f_{y}$ can be calculated linearly.

\section{$5 \quad$ Calibration with Simulated Data}

\subsection{Determining the Extrinsic Camera Parameters in the Classical PnP Problem with Simulated Data}

For this experiment, the calibration block was a regular hexagonal prismoid. The intrinsic camera parameters were known: $f_{x}=1000, f_{y}=1000, s=2$, $u_{0}=400, v_{0}=300$. The extrinsic camera parameters were set to $\mathrm{R}^{\prime}=[-0.0301,0.9852,-0.0026 ;-0.7125,-0.0185,0.7127 ; 0.7114,0.0193,0.7063]$, $\mathrm{t}=[-350 ;-250 ; 450]$,

Based on Section 3.1, the results were as follows:

$R^{\prime}=[-0.0301,0.9852,-0.0026 ;-0.7125,-0.0185,0.7127 ; 0.7114,0.0193,0.7063]$, $t^{\prime}=[-348.96 ;-251.62 ; 448.54]$ 


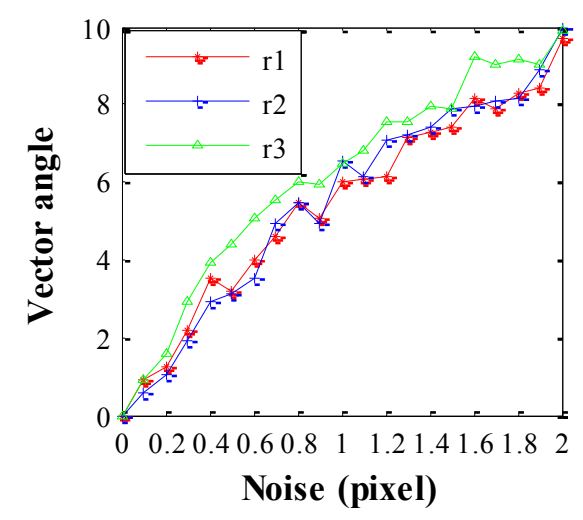

(a) Change curve of angle between the column vectors with noise in rotation matrix

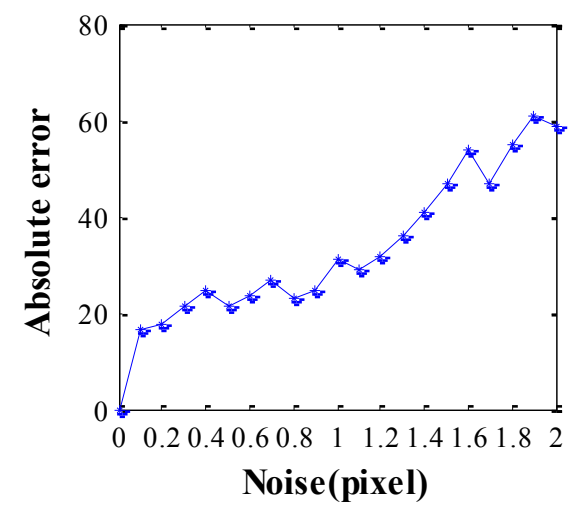

(b) Change curve of the absolute error of the maximum component with noise in translation vector

Figure 2 The change curves of the absolute error with respect to noise between the simulation results and the theoretical value of the extrinsic camera parameters.

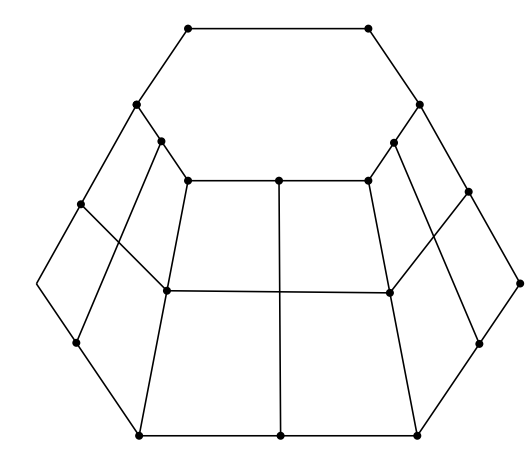

Figure 3 Simulated experimental image of a regular hexagonal prismoid.

To verify the credibility of the experimental results, we added uniform noise with amplitudes of $0-2$ pixels and an interval of 0.1 pixels to the image. The change curves of the absolute error with respect to noise between the simulation results and the theoretical value of the extrinsic camera parameters are shown in Figure 2(a) and Figure 2(b).

\subsection{Determining the Intrinsic and Extrinsic Camera Parameters in the PnP Problem with Simulated Data}

In the simulated experiment, the calibration block was a regular hexagonal prismoid (see Figure 3), and the camera settings were as follows: 


$$
f_{x}=1000, f_{y}=800, u_{0}=400, v_{0}=300
$$

The intrinsic parameters were set to:

$\mathrm{R}=[-0.9985,-0.0432,0.0331 ;-0.0317,-0.0325,-0.9990 ; 0.0442,-0.9985,0.03104]$, $t=[25 ; 40 ;-15]$

Based on Section 4.2, the results were as follows:

$f_{x}=1001.0763, f_{y}=800.4892, u_{0}=400.8563, v_{0}=299.7841$

$R^{\prime}=[-0.9972,-0.0351,0.0426 ;-0.0336,-0.0241,-0.9925 ; 0.0421,-0.9928,0.03851]$,

$t^{\prime}=[26.4533 ; 38.4729 ;-12.4386]$

To verify the test result, we added uniform noises to the image data, with amplitudes of $0-2$ pixels and an interval of 0.1 pixels. The change curves of the camera parameters with noise are shown in Figure 4.

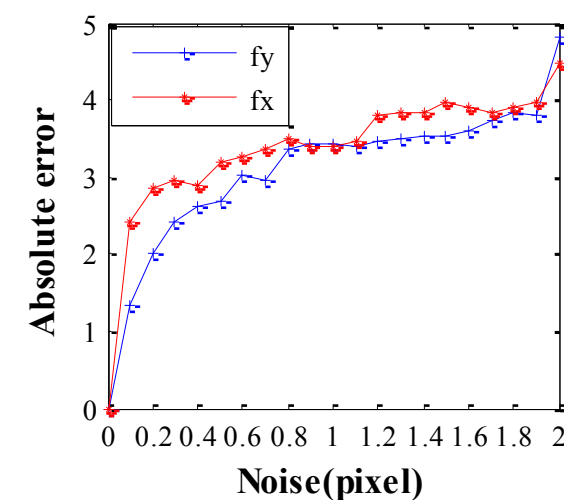

(a) $f_{x}, f_{y}$ change curve with noise

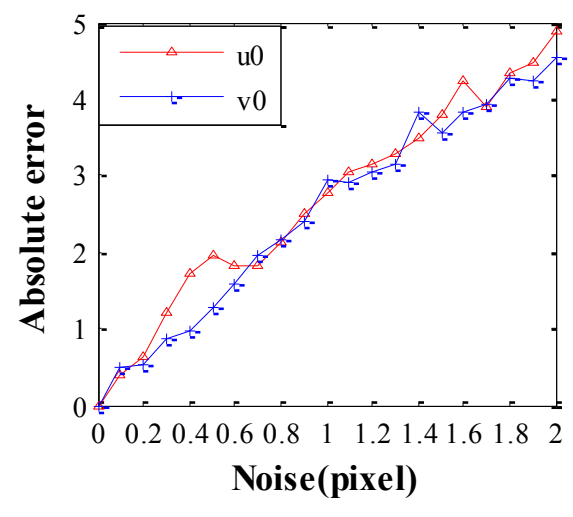

(b) $u_{0}, v_{0}$ change curve with noise

Figure 4 Intrinsic camera parameter change curves with noise.

\section{Calibration Using Real Data}

\subsection{Solving for the Extrinsic Camera Parameters in the PnP Problem with Real Data}

We took a regular hexagonal prismoid as the calibration block in the experiment, in which the length of the upper side was $5 \mathrm{~cm}$. In the experiment, the camera intrinsic parameters were known to be 
$K=[715.8783,2.4353,238.6983 ; 0,734.9895,317.2467 ; 0,0,1]$

A picture was taken of the regular hexagonal prismoid, as shown in Figure 5.

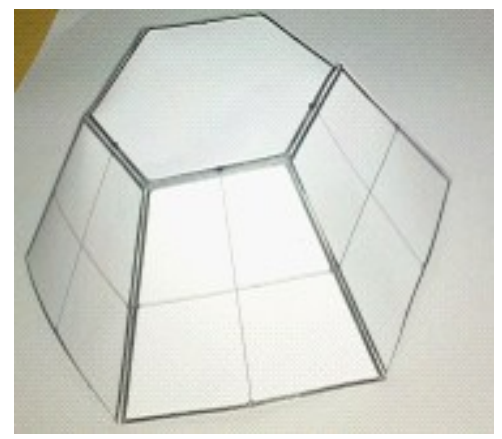

Figure 5 Image of a regular hexagonal prismoid.

The image resolution was set to $640 \times 480$. The upper surface of the regular hexagonal prismoid was taken as the $O_{w} X_{w} Y_{w}$ plane and one vertex of the upper surface as the origin in the WCS. Four feature points of the upper surface were chosen and the experiment was performed fifty times to obtain the average values of the extrinsic parameters:

$R=[0.9525,-0.0238,0.3035 ; 0.1986,-0.7069,-0.6788 ; 0.2307,0.7069,-0.6686]$, $T=[23.6324,-85.2341,42.5216]$

Professional equipment was required to obtain the extrinsic camera parameters, but being limited by the conditions, we could not know the truth value of the rotation matrix and the translation vector of the camera coordinate system and the world coordinate system. Therefore, we could not verify the correctness of all the parameters:

$$
\begin{aligned}
& R=[-0.0222,0.1524,-0.9881 ; 0.0300,0.9880,0.1517 ; 0.9993,-0.0263,-0.0265], \\
& t=[120.4518,-207.3245,-64.7721]
\end{aligned}
$$

Table 1 Intrinsic Camera Parameters for This Paper, DLT and Zhang.

\begin{tabular}{ccccc}
\hline & $f_{x}$ & $f_{y}$ & $u_{0}$ & $v_{0}$ \\
\hline This paper & 949.0972 & 761.3258 & 384.8921 & 264.0014 \\
DLT [13] & 942.8529 & 758.5289 & 381.5215 & 255.9859 \\
Zhang [11] & 948.5367 & 759.1364 & 386.2448 & 268.7401 \\
\hline
\end{tabular}


The truth value of the rotation matrix and translation vector between the CCS and the WCS cannot be accurately established with real data. The ground truth values of the camera parameters are not known, but the approach from [11] can be applied preceding experiments, using a calibration pattern that serves as a reference. To validate the feasibility and precision of the algorithm in this study, direct linear transformation (DLT) [13] was performed and the intrinsic parameters determined by DLT, Zhang [11], and our study were compared under the same conditions. The calibration results were as shown in Table 1. As seen in Table 1, we found that the calibration results using each of the three methods are similar to one another. Through our method, the intrinsic camera parameters can also be accurately determined.

\section{Conclusion}

In this study, we have taken a regular hexagonal prismoid as a calibration block and used the unique solution to classical PnP problems, where 4 control points are coplanar, and the uncalibrated $\mathrm{P} 4 \mathrm{P}$ problem. By combining this information with a corresponding relation between spatial points and image points to obtain a set of linear equations, the intrinsic and extrinsic camera parameters could be determined. We used the scene information of a regular prismoid to implement affine and metric reconstruction and analysed the experimental results. Both the simulation and the real data show that the method proposed in this paper is simple and has a high precision.

\section{Acknowledgements}

This research was supported by the Natural Foundation of Yunnan Province, China (2011FB017).

\section{References}

[1] Fisher, M.A. \& Bolles, R.C., Random Sample Consensus: A Paradigm for Model Fitting with Applications to Image Analysis and Automated Cartography, Communications of the ACM, 24(6), pp. 381-395, 1981.

[2] Harahck, R.M., Lee, D., Ottenburg, K. \& Nolle, M., Analysis and Solution of The Three Point Perspective Pose Estimation Problem, Proceedings of IEEE Conference on Computer Vision and Pattern Recognition, Germany, 3-6 June 1991.

[3] Horaud, R., Conio, B. \& Leboulleux, O., An Analytic Solution for The Perspective 4-Point Problem, Computer Vision, Graphics, and Image Processing, 47(1), pp. 33-44, 1989.

[4] Semple, J.G. \& Kneebone, G.T. Algebraic Projective Geometry, Oxford Univ Press, 1952. 
[5] Zhao, L.G., Wu, G.K. \& Ning, J.F. A Camera Calibration Method Based On Two Orthogonal Vanishing Points, Concurrency and Computation: Practice and Experience, 24(7), pp.1185-1199, 2013.

[6] Hu, Z.Y., Lei, C. \& Wu, F.C., A Short Note on P4P Problem, Acta Automatica Sinica, 27(6), pp. 770-776, 2001.

[7] Arsenovic, A., Chen, L.H., Bauwens, M.F., Li, H.L., Barker, N.S. \& Weikle, R.M., An Experimental Technique for Calibration Uncertainty Analysis, IEEE Transactions on Microwave Theory and Techniques, 61(1), 2013.

[8] Wu, F.C. \& Hu Z.Y., A Note on the P5P Problem with the Uncalibrated Camera, Chinese Journal of Computers, 24(11), pp. 1321-1326, 2001.

[9] Yuo, Y. \& Xu, X.H., An Analytic Solution for the P5P Problem with an Uncalibrated Camera, Chinese Journal of Computers, 30(7), pp. 11951200, 2007.

[10] Samper, D., Santolaria, J., Brosed, F.J., Majarena, A.C. \& Aguilar J.J., Analysis of Tsai Calibration Method Using Two- and Three-Dimensional Calibration Objects, Machine Vision and Applications, 24(1), pp.117131, 2013.

[11] Zhang, Z., A Flexible New Technique for Camera Calibration, IEEE Transaction on Pattern Analysis and Machine Intelligence, 22(11), pp. 1330-1334, 2000.

[12] Wu F.C., Mathematical Methods in Computer Vision, Science Press, 2008.

[13] Hartley, R. \& Zisserman, A., Multiple View Geometry in Computer Vision, Cambridge, 2000. 